\title{
Cellulose Dimethylphenylcarbamate-bonded Carbon-clad Zirconia for Chiral Separation in High Performance Liquid Chromatography
}

\author{
Sang Hyun Kwon, ${ }^{11}$ Yoshio OKamoto, ${ }^{2}$ Chiyo Yamamoto, ${ }^{* 3}$ Wonjo Cheong, ${ }^{* 4}$ \\ Myeonghee MoON, ${ }^{* 5}$ and Jung Hag PARK ${ }^{* 1 \dagger}$ \\ *1 Department of Chemistry, Yeungnam University, Gyeongsan 712-749, Korea \\ *2 EcoTopia Science Institute, Nagoya University, Nagoya 464-8603, Japan \\ *3 Department of Applied Chemistry, Graduate School of Engineering, Nagoya University, Nagoya 464-8603, Japan \\ *4 Department of Chemistry, Inha University, Incheon 402-751, Korea \\ *5 Department of Chemistry, Yonsei University, Seoul 120-749, Korea
}

\begin{abstract}
Porous zirconia particles are very robust material and have received considerable attention as a stationary phase support for HPLC. We prepared cellulose dimethylphenylcarbamate-bonded carbon-clad zirconia (CDMPCCZ) as a chiral stationary phase (CSP) for separation of enantiomers of a set of 14 racemic compounds in normal phase (NP) and reversed-phase (RP) liquid chromatography. Retention and enantioselectivity on CDMPCCZ were compared to those on CDMPC-coated zirconia (CDMPCZ) to see how the change in immobilization method of the chiral selector affects the retention and chiral selectivity. In NPLC, retention was longer and the number of resolved racemates was smaller on CDMPCCZ than on CDMPCZ. However, chiral selectivity factors for some resolved racemates were better on CDMPCCZ than on CDMPCZ. The longer retention on CDMPCCZ is likely due to strong, non-chiral discriminating interactions with the carbon layer on CDMPCZ. In RPLC only two racemates were resolved on CDMPCCZ, but retention times were shorter than, and resolutions were comparable to, those in NPLC, indicating a potential for improving chromatographic performance of the CDMPCCZ column in RPLC with optimized column preparation and separation conditions.
\end{abstract}

(Received June 5, 2006; Accepted August 28, 2006; Published December 10, 2006)

HPLC based on chiral stationary phases (CSPs) has become one of the most attractive approaches to chiral separations, due to their simplicity for determining optical purity and easy extension to the semipreparative and preparative scales. ${ }^{1}$ One of the major problems in using many CSPs is their narrow range of analyte applicability; they can only discriminate a limited number of specific types of chemical entities, and it is frequently necessary to derivatize the compounds of interest to achieve separation. ${ }^{2}$ On the other hand, the polysaccharide derivative-based CSPs developed by Okamoto and coworkers ${ }^{3-7}$ have proven to be highly versatile and rugged. Okamoto reported that the resolution of 483 racemic mixtures on cellulose and that $80 \%$ of them were successfully resolved on either the cellulose or amylose tris(3,5-dimethylphenylcarbamate) (CDMPC or ADMPC). ${ }^{8}$

Silica is the most popular choice for support of HPLC stationary phase ligands due to the mechanical strength, wide range of particle and pore dimensions, pore structure and wellestablished silane chemistry. However, silica and bonded phase ligands have stability problems. Silica dissolves in mobile phase buffered at or above $\mathrm{pH} 8$ with loss of bonded phase ligand and column packing. ${ }^{2}$ Loss of organosilanes from the silica surface via hydrolysis proceeds rapidly at low $\mathrm{pH}(<3)$

$\dagger$ To whom correspondence should be addressed.

E-mail: jhpark@ynu.ac.kr and at high temperature $\left(\geq 40^{\circ} \mathrm{C}\right)$. These deficiencies of the column packing create problems of poor injection reproducibility, poor peak shape, and high backpressure, thus making method development tasks difficult. Siloxane-bonded silica phases with improved hydrolytic stability at extreme $\mathrm{pH}$ for use in reversedphase liquid chromatography were introduced. ${ }^{10-13}$ However, careful choices of operating conditions, such as the use of acetonitrile instead of methanol, the use of boric acid or organic buffers in low concentration and low temperature, are still required to achieve acceptable column lifetimes. ${ }^{14-17}$

Zirconia particles are very robust material; they show no detectable signs of dissolution over the $\mathrm{pH}$ range from 1 to 14 and have been used for prolonged periods at temperatures up to $200^{\circ} \mathrm{C}$ in chromatographic separations. Thus, zirconia has received considerable attention as a stationary phase support for HPLC over the last decade. ${ }^{18,19}$ One of us has been working to develop efficient and chemically stable CSPs on zirconia substrates. ${ }^{20-23}$ Bare zirconia cannot be covalently modified like silica due to the instability of $\mathrm{Zr}-\mathrm{C}$ and $\mathrm{Zr}-\mathrm{O}-\mathrm{Si}$ bonds in water. ${ }^{24}$ Zirconia-based CSPs reported have thus been prepared by coating chiral selectors on zirconia surface by utilizing Lewis acid-base chemistry. Recently we reported chiral separation of $\mathrm{N}$-(2,4-dinitrophenyl) (DNP) amino acids on a 9$O$-(phenyloxycarbonyl)quinine bonded carbon-clad zirconia (QNOCZ) in reversed-phase liquid chromatography (RPLC). ${ }^{25}$ Carbon-clad zirconia is made by passing organic vapors over 
very hot porous zirconia. ${ }^{26}$ Carbon-clad zirconia particles show similar mechanical, thermal and chemical stability to bare zirconia particles but no appreciable Lewis acidity. They do not exhibit peak tailing for amines nor do they adsorb phosphates or carboxylates. ${ }^{27}$

While most polysaccharides-coated silica (or zirconia) CSPs are durable in normal-phase eluents such as hexane-2-propanol mixtures, cellulose tris(3,5-dichlorophenylcarbamate) is soluble in hexane-2-propanol mixtures containing more than $10 \%$ of 2 propanol and in some other solvents such as tetrahydrofuran and chloroform. The polysaccharide derivatives were chemically bonded to silica to avoid this problem. ${ }^{28}$ In this work, we report preliminary results obtained with 3,5-dimethylphenylcarbamate of cellulose regioselectively bonded to carbon-clad zirconia particles (CDMPCCZ) as chiral stationary phase for HPLC. Oliveros et al. immobilized CDMPC bearing 10-undeceno groups onto graphite by thermal treatment in the presence of azoisobutyronitrile. ${ }^{29}$ However, CDMPC moieties were fixed by crosslinking but were not directly bonded to the graphite surface. Chromatographic performance of CDMPCCZ was examined by measuring the chiral discrimination of a set of 14 racemic compounds on the column in normal phase (NP) and reversed-phase (RP) liquid chromatography. The chiral selectivity of CDMPCCZ was compared to that on cellulose tris(3,5-dimethylphenylcarbamate)-coated zirconia (CDMPCZ) to see how the change in the immobilization of the chiral selector and the type of the base material (carbon-clad vs. bare zirconia) affects the retention and chiral selectivity.

\section{Experimental}

\section{Reagents and materials}

All reagents used for the preparation of the stationary phase were reagent grade or better. Cellulose (Avicel) was purchased from Merck (Darmstadt, Germany). Triphenylmethyl chloride and 4,4'-diphenylmethane diisocyanate were of reagent grade from TCI (Tokyo, Japan). 3,5-Dimethylphenyl isocyanate, tetrahydrofuran and pyridine were obtained from Aldrich (Milwaukee, USA). Carbon-clad zirconia in spherical shape, particle diameter of $5 \mu \mathrm{m}$, pore size of $30 \mathrm{~nm}$ and surface area of $30 \mathrm{~m}^{2} / \mathrm{g}$, were obtained from ZirChrom Separations (Anoka, MN, USA). Water was processed with an Elgastat UHQ water purification system (Bucks, UK). 2-Propanol was HPLC grade (J. T. Baker, Phillipsburg, USA). n-Hexane was purchased from EM Sciences (Gibbstown, USA). All other chemicals were of the best quality available and were used as received without any further purification. The racemic compounds studied are shown in Fig. 1. All are commercially available. Solutions at a concentration of $0.1 \mathrm{mg} / \mathrm{mL}$ were prepared by dissolving the compounds in the mobile phase.

\section{Preparation of CDMPC-coated zirconia}

Cellulose tris(3,5-dimethylphenylcarbamate) was synthesized as previously reported ${ }^{5}$ and characterized by elemental analyses, IR and NMR spectroscopy. Typically, $1.0 \mathrm{~g}$ of dehydroxylated bare zirconia particles were suspended in $10 \mathrm{~mL}$ of THF and sonicated under vacuum for $15 \mathrm{~min}$ to eliminate the air from the pores. Polymer loading of $4 \%$ by weight was chosen since this loading has been shown to offer excellent chiral recognition ability and column efficiency. The corresponding amount of CDMPC was dissolved in $10 \mathrm{~mL}$ of THF and the solution was added to the slurry of zirconia in THF using a syringe pump at a rate of $0.04 \mathrm{~mL} / \mathrm{min}(\sim 4 \mathrm{~h})$. The suspension was stirred overnight and then the solvent was slowly removed by rotary evaporation at room temperature. Finally, the particles were dried in vacuum at $50^{\circ} \mathrm{C}$.

\section{Preparation of CDMPC-bonded carbon-clad zirconia}

The CDMPC-bonded carbon-clad zirconia CSP was prepared by the reaction of a cellulose derivative and 4-hydroxyphenylbonded carbon-clad zirconia with 4,4'-diphenylmethane diisocyanate. The cellulose derivative was regioselectively bonded to carbon-clad zirconia on its glucose unit.

\section{Preparation of 4-hydroxyphenyl carbon-clad zirconia}

4-Hydroxyphenyl-bonded carbon-clad zirconia was prepared according to a procedure in the literature ${ }^{30}$ with modification. Briefly, $1 \mathrm{~g}$ of carbon-clad zirconia was added to a solution of $0.22 \mathrm{~g}$ of 4-aminophenol dissolved in a mixture of $4 \mathrm{~mL}$ of ethanol and $8 \mathrm{~mL}$ of water. The resulting suspension was cooled to $30^{\circ} \mathrm{C}$ and $0.13 \mathrm{~mL}$ of concentrated nitric acid was added. An aqueous solution containing $0.14 \mathrm{~g}$ of sodium nitrite was then added gradually with stirring, forming 4hydroxybenzene diazonium hydroxide inner salt in situ, which reacts with the carbon on zirconia. The resulting product, 4hydroxyphenyl-bonded carbon-clad zirconia, was filtered, washed three times each with water, ethanol and acetone, and dried in an oven at $125^{\circ} \mathrm{C}$. The bonding density of 4hydroxyphenyl groups was found from microanalysis to be 4.80 $\mu \mathrm{mol} \mathrm{m}{ }^{-2}$ based on the percent of hydrogen.

Preparation of cellulose 2,3-bis(3,5-dimethylphenylcarbamate)bonded carbon-clad zirconia

Cellulose 2,3-bis(3,5-dimethylphenylcarbamate) was prepared according to a procedure in the literature. ${ }^{31}$ Cellulose 2,3bis(3,5-dimethylphenylcarbamate) was dissolved in $15 \mathrm{~mL}$ of THF and the solution was coated on 4-hydroxyphenyl carbonclad zirconia. After THF had been evaporated in vacuo, the cellulose-coated particles were dispersed in a mixture of $10 \mathrm{~mL}$ of dry toluene and $2 \mathrm{~mL}$ of dry pyridine that contained $25.8 \mathrm{mg}$ of 4,4'-diphenylmethane diisocyanate, and the mixture was stirred for $5 \mathrm{~h}$ at $90^{\circ} \mathrm{C}$. An excess of 3,5-dimethylphenyl isocyanate was added and the mixture was allowed to react with the remaining hydroxyl groups at the 6-position at $90^{\circ} \mathrm{C}$ for $24 \mathrm{~h}$. The CDMPC-bonded carbon-clad zirconia particles thus obtained were collected by filtration and washed with THF to remove unbound $\mathrm{CDMPC}$, and dried in an oven at $125^{\circ} \mathrm{C}$. Weight percent of chiral selector on zirconia, based on the percent of hydrogen obtained from microanalysis, was found to be $3.2 \%$.

\section{Chromatography}

Packing materials were suspended in a hexane/2-propanol (1:1) mixture and packed into $20 \mathrm{~cm} \times 1 \mathrm{~mm}$ (i.d.) columns using the downward slurry method at $c a .7000$ psi. 2-Propanol was employed as the displacing solvent. The chromatographic system was consisted of a Model 7520 injector with a $0.5-\mu \mathrm{L}$ internal loop (Rheodyne, CA, USA), a Model 530 column oven (Alltech, IL, USA) set at $25^{\circ} \mathrm{C}$ and a Linear Model $200 \mathrm{UV} / \mathrm{VIS}$ detector (Alltech) with a $0.25-\mu \mathrm{L}$ flowcell set at $254 \mathrm{~nm}$. A Hewlett-Packard (Avondale, CA, USA) Series 3365 integrating recorder was used to record chromatograms. The mobile phases were mixtures of hexane and 2-propanol (98:2 or 90:10 v/v\%), acetonitrile and water $(70: 30 \mathrm{v} / \mathrm{v} \%)$, and methanol and water $(85: 15 \mathrm{v} / \mathrm{v} \%)$. They were filtered through a membrane filter of $0.45-\mu \mathrm{m}$ pore size and degassed prior to use. The flow rate was $100 \mu \mathrm{L} / \mathrm{min}$. The dead times were estimated by using 1,3,5-tritert-butylbenzene and $\mathrm{D}_{2} \mathrm{O}$ as unretained compounds in NPLC ${ }^{32}$ and RPLC, respectively. 


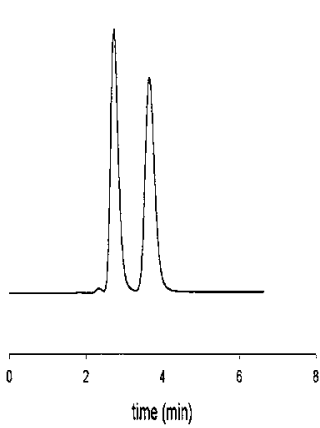

(a)

Fig. 1 Separation of (a) trans-stilbene oxide on CDMPCZ in 98:2 (v/v\%) $n$-hexane/2-propanol, (b) trans-stilbene oxide on CDMPCCZ in 98:2 (v/v\%) n-hexane/2-propanol, and (c) 1-phenyl-1propanol on CDMPCCZ in 90:10 (v/v\%) methanol/water.

\section{Results and Discussion}

\section{NPLC separation on CDMPC-bonded carbon-clad zirconia}

Retention $(k)$ and selectivity factors $(\alpha)$ of 14 racemic compounds on CDMPCCZ in 98:2 (v/v) and 90:10 (v/v) hexane/2-propanol eluents are listed in Table 1 along with the data obtained on CDMPCZ. In 98:2 hexane/2-propanol, 8 racemic compounds were separated on CDMPCCZ while 11 compounds were separated on CDMPCZ. In 90:10 hexane/2propanol, 6 racemates were resolved on CDMPCCZ while 9 racemates were resolved on CDMPCZ. Retention factors of the racemic compounds studied are much longer and peaks are broader on CDMPCCZ than on CDMPCZ even if the polymer load is lower on CDMPCCZ (3.2 w/w\%) than on CDMPCZ (4.0 $\mathrm{w} / \mathrm{w} \%)$. A chromatogram for the resolution of trans-stilbene oxide on CDMPCCZ in 98:2 (v/v) hexane/2-propanol is shown in Fig. 1b. The peaks are tailed and broader than those observed on CDMPCZ (Fig. 1a).23

The longer retention on CDMPCCZ is very likely due mainly to strong non-stereodiscriminating interactions of the solute molecule with the carbon layer on the zirconia surface and, to a much smaller extent to extra dipolar interactions of the solute molecule with the phenyl(4,4-diphenylmethane)dicarbamoyl group at the 6-position of the glucose unit of CDMPC in the linkage between the CDMPC moiety and the carbon layer of the zirconia. Dispersive and dipole (the polar functional group of the solute molecule)-induced dipole (the carbon layer on zirconia) interactions may prolong retention to a great extent. The phenyl(4,4-diphenylmethane)dicarbamoyl moiety in the linkage on CDMPCCZ, which replaces the 6-position 3,5dimethylphenylcarbamoyl group on CDMPCZ, may also undergo additional dipolar and hydrogen bonding (HB) interactions with dipolar and hydrogen bond-accepting functionality of the solute molecule, thereby increasing retention.

Chiral discrimination ability of CDMPCCZ is not as good as that of CDMPCZ. It has been reported with silica as the support material that the chiral recognition abilities of nonregioselectively bonded polysaccharides CSPs were usually decreased by immobilization, yielding a lower resolving ability than those for the coated-type CSPs. Chiral selectivity factors are better for 4 (compounds $2, \mathbf{4 , 8}$ and 11) out of 8 racemates resolved in 98:2 hexane/2-propanol, and for 2 (compounds 4 and 11) out of 6 racemates in 90:10 hexane/2-propanol on CDMPCCZ than those on CDMPCZ. The lower chiral selectivity for CDMPCCZ is likely due to the lower column efficiency of CDMPCCZ than that for CDMPCZ. Plate numbers of the two CSP columns computed for the unretained marker in 98:2 (v/v) hexane/2-propanol were 1122 and 2164 for CDMPCCZ and CDMPCZ, respectively. Additional reasons for the lower chiral selectivity for CDMPCCZ are as follows: the lower load of the chiral selector (3.2\% vs. 4.0\%) and strong, non-chiral discriminating interactions of the carbon layer of the CSP with the racemate molecules, which would slow down molecular transfer processes between the stationary and mobile phase. We do not have a clear explanation for the better chiral selectivity obtained for the 4 racemic compounds mentioned above (compounds $\mathbf{2 , 4 , 8}$ and 11) in 98:2 hexane/2-propanol. It is thought that the extra dipolar and hydrogen bonding (HB) interactions of the phenyl(4,4-diphenylmethane)dicarbamoyl moiety in the linkage on CDMPCCZ with dipolar and/or hydrogen bond-accepting moieties of the solute molecule might have contributed to increased chiral recognition for the compounds.

\section{RPLC separation on CDMPC-bonded carbon-clad zirconia}

It is expected for CDMPCCZ to show a typical RP behavior in reversed-phase mobile phases because CDMPCCZ is essentially an RP phase on which a small mass of CDMPC polymer is anchored on the carbon phase that covers zirconia. Only two compounds (4 and 11) were separated in two different RP mobile phases (Table 1). On CSPs with cellulose derivatives having 2,3-O-acetyl and benzoyl substituents, better chiral separation was obtained for a set of racemic compounds similar to those in this study in NPLC eluent (95:5 hexane/2-propanol) than in RPLC eluent (70:30 methanol/water). ${ }^{33}$ A chromatogram for the resolution of 1-phenyl-1-propanol on CDMPCCZ in 90:10 (v/v \%) methanol/water is shown in Fig. 1c. Retention times are shorter, but peaks are better separated and more symmetrical than those observed in the NPLC separation.

As CDMPCCZ was believed to be stable in mobile phases of alkaline $\mathrm{pH}$, we attempted chiral separation of compounds $\mathbf{8}$ and 9 and ten other basic racemic compounds in 70:30 methanol/triethylamine acetate buffer $(0.02 \mathrm{M}, \mathrm{pH} 10)$. We did not attempt to optimize the mobile phase composition ( $\mathrm{pH}$, type and content of organic modifier) for the separation. Since enantiomers of basic compounds would be less protonated at $\mathrm{pH}$ 10 and thus more retained, it was expected that one would obtain resolution for at least some of the basic racemates tested in this alkaline eluent. Disappointingly, none were resolved in that eluent.

In summary, cellulose dimethylphenylcarbamate-bonded carbon-clad zirconia was prepared as a chiral stationary phase for separation of enantiomers of a set of racemic compounds. 
Table 1 Chromatographic data on CDMPCCZ and CDMPCZ

\begin{tabular}{|c|c|c|c|c|c|c|c|c|c|c|c|c|}
\hline \multirow{3}{*}{$\begin{array}{l}\text { Compound } \\
\text { No. }^{\text {a }}\end{array}$} & \multicolumn{4}{|c|}{$k_{1}^{\mathrm{b}}$} & \multicolumn{4}{|c|}{$\alpha^{\mathrm{c}}$} & \multicolumn{4}{|c|}{$R_{\mathrm{s}}^{\mathrm{d}}$} \\
\hline & \multicolumn{2}{|c|}{$\mathrm{CDMPCCZ}^{\mathrm{e}}$} & \multicolumn{2}{|c|}{$\mathrm{CDMPCZ}^{\mathrm{f}}$} & \multicolumn{2}{|c|}{$\mathrm{CDMPCCZ}^{\mathrm{e}}$} & \multicolumn{2}{|c|}{$\mathrm{CDMPCZ}^{\mathrm{f}}$} & \multicolumn{2}{|c|}{$\mathrm{CDMPCCZ}^{\mathrm{e}}$} & \multicolumn{2}{|c|}{$\mathrm{CDMPCZ}^{\mathrm{f}}$} \\
\hline & $98: 2$ & $90: 10$ & $98: 2$ & $90: 10$ & $98: 2$ & $90: 10$ & $98: 2$ & $90: 10$ & $98: 2$ & $90: 10$ & $98: 2$ & $90: 10$ \\
\hline 1 & 38.09 & 6.48 & 9.93 & 1.50 & 1.51 & 1.37 & 3.46 & 2.94 & 1.93 & 0.55 & - & - \\
\hline 2 & 10.93 & 2.56 & 9.24 & 1.99 & 1.14 & 1.00 & 1.10 & 1.00 & 0.66 & - & - & - \\
\hline 3 & 20.81 & 4.36 & 6.12 & 1.43 & 1.21 & 1.17 & 1.94 & 1.88 & 0.83 & 0.41 & - & - \\
\hline \multirow[t]{2}{*}{4} & 3.88 & 1.34 & 3.30 & 0.62 & 1.73 & 1.35 & 1.37 & 1.14 & 1.88 & 0.77 & - & - \\
\hline & $(0.86)^{\mathrm{g}}$ & $(0.30)^{\mathrm{h}}$ & $(0.19)^{\mathrm{g}}$ & $(0.07)^{\mathrm{h}}$ & $(1.97)^{g}$ & $(7.87)^{\mathrm{h}}$ & $(1.63)^{\mathrm{g}}$ & $(2.88)^{\mathrm{h}}$ & $(1.86)^{\mathrm{g}}$ & $(1.20)^{\mathrm{h}}$ & - & - \\
\hline 5 & 4.86 & 1.51 & 2.59 & 1.06 & 1.00 & 1.00 & 1.15 & 1.00 & - & - & - & - \\
\hline 6 & 8.24 & 1.70 & 5.69 & 1.27 & 1.18 & 1.11 & 1.21 & 1.14 & 0.49 & 0.37 & - & - \\
\hline 7 & 4.14 & 1.17 & 2.65 & 0.82 & 1.00 & 1.00 & 1.17 & 1.00 & - & - & - & - \\
\hline 8 & 4.66 & 2.31 & 2.58 & 0.74 & 1.21 & 1.22 & 1.17 & 1.50 & 0.46 & 0.43 & - & - \\
\hline 9 & $\mathrm{NE}^{\mathrm{i}}$ & 100.4 & $\mathrm{NE}^{\mathrm{i}}$ & 7.82 & - & 1.00 & - & 1.30 & - & - & - & - \\
\hline 10 & 2.24 & 1.68 & 1.30 & 0.35 & 1.27 & 1.00 & 2.83 & 2.23 & 0.73 & - & - & - \\
\hline \multirow[t]{2}{*}{11} & 4.37 & 2.94 & 1.31 & 0.62 & 7.07 & 4.07 & 4.05 & 3.75 & 2.83 & 2.76 & - & - \\
\hline & $(1.25)^{\mathrm{g}}$ & $(1.03)^{\mathrm{h}}$ & $(\mathrm{NR})^{\mathrm{g}}$ & $(\mathrm{NR})^{\mathrm{h}}$ & $(2.26)^{\mathrm{g}}$ & $(2.71)^{\mathrm{h}}$ & - & - & $(1.63)^{\mathrm{g}}$ & $(1.58)^{\mathrm{h}}$ & - & - \\
\hline 12 & 26.65 & 10.54 & 6.68 & 3.21 & 1.00 & 1.00 & 1.00 & 1.00 & - & - & - & - \\
\hline 13 & 111.56 & 35.75 & 9.83 & 3.40 & 1.00 & 1.00 & 1.00 & 1.00 & - & - & - & - \\
\hline 14 & 3.79 & 2.23 & 1.27 & 0.53 & 1.00 & 1.00 & 1.31 & 1.30 & - & - & - & - \\
\hline
\end{tabular}

a. Hexane/2-propanol was used as the eluent unless stated otherwise. Racemic compounds studied: 2,2,2-trifluoro-1-(9-anthryl) ethanol (1), $\alpha$-(trifluoromethyl)benzyl alcohol (2), $\alpha$-methyl-1-naphthalenemethanol (3), 1-phenyl-1-propanol (4), 1-phenyl-2-propanol (5), 3phenyl-1-butanol (6), 1-phenyl-1-butanol (7), Tröger's base (8), (3,5-dinitrobenzoyl)- $\alpha$-methylbenzylamine (9), trans-stilbene oxide (10), 4-phenyl-1,3-dioxane (11), $\gamma$-phenyl- $\gamma$-butyrolactone (12), $\gamma$-(2-naphthyl)- $\gamma$-butyrolactone (13), and $\alpha$-methylbenzyl cyanide (14). b. Retention factor of the first eluting enantiomer. c. Selectivity factor. d. Resolution. e. Carbon-clad zirconia particles: spherical shape, diameter, $5 \mu \mathrm{m}$; pore size, $30 \mathrm{~nm}$; surface area, $30 \mathrm{~m}^{2} / \mathrm{g}$. Column dimension, $20 \mathrm{~cm}$ (length) $\times 1 \mathrm{~mm}$ (i.d.); polymer load, 3.2\% (w/w). Plate number $(N)$ calculated from the unretained 1,3,5-tri-tert-butylbenzene peak $=1122$. f. Zirconia particles: spherical shape, diameter, $5 \mu \mathrm{m}$; pore size, $30 \mathrm{~nm}$; surface area, $30 \mathrm{~m}^{2} / \mathrm{g}$. Column dimension, $30 \mathrm{~cm}$ (length) $\times 1 \mathrm{~mm}$ (i.d.); polymer load, $4.0 \%$ (w/w). $N$ for 1,3,5tri-tert-butylbenzene peak $=2164$. The $k_{1}$ and $\alpha$ values were taken but $R_{\mathrm{s}}$ values were not available from the literature. ${ }^{23} \mathrm{~g}$. Mobile phase was 70:30 (v/v\%) acetonitrile/water. $\mathrm{NR}=$ not resolved. h. Mobile phase was 85:15 (v/v\%) methanol/water. i. Not eluted in $3 \mathrm{~h}$.

Retention and enantioselectivity on CDMPCCZ were compared to those on CDMPC-coated zirconia. In NPLC, retention was longer and chiral selectivities were in general lower, and the number of racemates resolved was smaller on CDMPCCZ than on CDMPCZ. The longer retention and lower selectivity of CDMPCCZ is likely due to strong, non-chiral discriminating interactions with the carbon layer on CDMPCZ. In view of the fact that the NPLC chiral selectivity for CDMPCZ was comparable to that for CDMPC-coated silica (CDMPCS), ${ }^{23}$ the performance for CDMPCCZ is not as good as those for the coated type CSPs. However, chiral selectivity factors for some resolved racemates were better on CDMPCCZ than CDMPCZ. In RPLC separation, only 2 racemates were resolved on CDMPCCZ. However, retention times were shorter than, but resolutions were comparable to, those in NPLC. It has been reported that the bonded cellulose phases are very stable even in chloroform in which the polysaccharide derivatives are dissolved or swollen. ${ }^{7,34}$ Even though the number of racemates resolved on CDMPCCZ is small, it is expected that the better stability of the bonded CSP may be an advantage that could be utilized in an endeavor for improving chromatographic performance of the CDMPCCZ column by optimizing the packing procedure for a column of a higher plate number and the mobile phase compositions for resolution of the specific racemates.

\section{Acknowledgements}

This work was supported in part by a grant (R01-2006-00010004-0) from the Korea Science and Engineering Foundation.

\section{References}

1. E. Francotte, in "Chiral Separations: Applications and Technology”, ed. S. Ahuja, 1997, Chap. 5, American Chemical Society, Washington.

2. J. Dingenen, in "A Practical Approach to Chiral Separations by Liquid Chromatography", ed. G. Subramanian, 1994, Chap. 6, VCH, New York.

3. Y. Okamoto, M. Kawashima, K. Yamamoto, and K. Hatada, Chem. Lett., 1984, 739.

4. Y. Okamoto, M. Kawashima, and K. Hatada, J. Am. Chem. Soc., 1984, 106, 5357.

5. Y. Okamoto, M. Kawashima, and K. Hatada, $J$. Chromatogr., 1986, 363, 173.

6. Y. Okamoto and E. Yashima, Angew. Chem., Int. Ed., 1998, 37, 1020.

7. C. Yamamoto and Y. Okamoto, Bull. Chem. Soc. Jpn., 2004, 77, 227.

8. Y. Okamoto, Y. Kaida, R. Aburatani, and K. Hatada, in "Chiral Separations by Liquid Chromatography", ed. S. Ahuja, 1991, ACS Symposium Series 471, American Chemical Society, Washington, D.C., $101-113$.

9. J. J. Kirkland, M. A. van Straten, and H. A. Claessans, J. Chromatogr., A, 1995, 691, 3.

10. J. J. Kirkland, J. L. Glajch, and R. D. Farlee, Anal. Chem., 1989, 61, 2.

11. K. D. Lork, K. K. Unger, and J. J. Kinkel, J. Chromatogr., 1986, 352, 199.

12. J. J. Kirkland and J. W. Henderson, J. Chromatogr. Sci., 1994, 32, 473. 
13. J. J. Kirkland, J. B. Adams, M. A. van Straten, and H. A. Claessens, Anal. Chem., 1998, 70, 4344.

14. J. Nawrocki, J. Chromatogr., A, 1997, 779, 29.

15. J. J. Kirkland, J. W. Henderson, J. J. DeStefano, M. A. van Straten, and H. A. Claessens, J. Chromatogr., A, 1997, 762, 97.

16. H. A. Claessens, M. A. van Straten, and J. J. Kirkland, J. Chromatogr., A, 1996, 728, 259.

17. J. J. Kirkland, M. A. van Straten, and H. A. Claessens, J. Chromatogr., A, 1998, 797, 111.

18. J. Nawrocki, C. J. Dunlap, P. W. Carr, and J. A. Blackwell, Biotechnol. Prog., 1994, 10, 561.

19. P. T. Jackson and P. W. Carr, Chemtech, 1998, Oct. 29, 29.

20. C. B. Castells and P. W. Carr, Anal. Chem., 1999, 71, 3013.

21. J. H. Park, J. K. Ryu, J. K. Park, C. V. McNeff, and P. W. Carr, Chromatographia, 2001, 53, 405.

22. S. Y. Park, J. K. Park, J. H. Park, C. V. McNeff, and P. W Carr, Microchem. J., 2001, 70, 179.

23. J. H. Park, Y. C. Whang, Y. J. Jung, Y. Okamoto, C. Yamamoto, P. W. Carr, and C. V. McNeff, J. Sep. Sci., 2003, 26, 1331.
24. J. Nawrocki, M. P. Rigney, A. McCormick, and P. W. Carr, J. Chromatogr., A, 1993, 657, 299.

25. J. H. Park, J. W. Lee, S. H. Kwon, J. S. Cha, P. W. Carr, and C. V. McNeff, J. Chromatogr., A, 2004, 1050, 151.

26. T. P. Weber, P. W. Carr, and E. F. Funkenbush, J. Chromatogr., 1990, 519, 31.

27. M. P. Rigney, T. P. Weber, and P. W. Carr, J. Chromatogr., 1989, 484, 273.

28. Y. Okamoto, R. Aburatani, S. Miura, and K. Hatada, J. Liq. Chromatogr., 1987, 10, 1613.

29. L. Oliveros, P. Lopez, C. Minguillion, and P. Franco, $J$. Liq. Chromatogr., 1995, 18, 1521

30. J. A. Belmont, U. S. Patent, 1997, 5672198.

31. E. Yahima, H. Fukaya, and Y. Okamoto, J. Chromatogr., A, 1994, 677, 11 .

32. H. Koller, K. H. Rimbock, and A. Mannschreck, J. Chromatogr., 1983, 282, 89.

33. N. Kasuya, J. Nakashima, T. Kubo, A. Sawatari, and N. Habu, Chirality, 2000, 12, 670.

34. P. Franco, C. Minguillon, and L. Oliveros, J. Chromatogr., A, 1998, 793, 239. 